\title{
Prostate cancer metastasis to the distal phalanx of the left hallux: The first confirmed case and literature review
}

\author{
XINBING SUI $^{1 *}$, YAN HU $^{2 *}$, CHENG ZHANG $^{3}$, HONGMING PAN $^{1}$ and DA LI ${ }^{1}$ \\ Departments of ${ }^{1}$ Medical Oncology and ${ }^{2}$ Pathology, Sir Run Run Shaw Hospital, \\ Zhejiang University, Hangzhou, Zhejiang 310016; ${ }^{3}$ Department of Medical Oncology, \\ The Affiliated Hospital of Hangzhou Normal University, Hangzhou, Zhejiang 310015, P.R. China
}

Received April 14, 2015; Accepted May 26, 2016

DOI: $10.3892 / \mathrm{ol} .2016 .4701$

\begin{abstract}
Prostate cancer is one of the most common carcinomas in Asia, as well as all over the world. The vast majority of prostate cancer patients present with bone metastases, which mainly involve the axial bones, with pain as the most common symptom. The present study reports the first case of prostate cancer metastasis to the hallux in a 73-year-old man who presented with pain and a swollen phalanx of the left hallux. This symptom developed gradually over a period of several months and could not be improved with the common treatments. Due to a pathological fracture, amputation of the left phalanx was performed. Notably, the immunohistochemical examinations of the surgical sample demonstrated the presence of a metastatic lesion from prostate cancer. The present study describes an unusual presentation involving phalangeal metastasis in a prostate cancer patient, and a systematic review of reported cases of rare metastatic events in prostate cancer is also discussed.
\end{abstract}

\section{Introduction}

Prostate cancer is the most common carcinoma in males around the world and the fifth most common cause of cancer-associated mortality. The majority of prostate cancer patients succumb to metastatic disease (1). Distant metastases are the most common cause of cancer-associated mortality in prostate cancer patients (2). The bone is a common site of metastasis in prostate cancer and this type of metastasis presents in $>80 \%$ of patients who succumb to prostate cancer (3). The bone metastatic lesions from prostate cancer show more

Correspondence to: Professor Hongming Pan or Dr Da Li, Department of Medical Oncology, Sir Run Run Shaw Hospital, Zhejiang University, 3 Qingchun East Road, Hangzhou, Zhejiang 310016, P.R. China

E-mail: drpanhm@aliyun.com

E-mail: da123@163.com

${ }^{*}$ Contributed equally

Key words: prostate cancer, bone metastases, terminal phalanx osteoblastic than osteolytic bone lesions compared with those that originate from other cancer types (4). Metastases to the bone mainly involve the axial bones, and prostate cancer rarely metastasizes to the distal phalanges. The current study reports the case of a patient with prostate cancer that metastasized to the distal phalanx of the left hallux. A systematic review of the associated literature regarding prostate cancer with non-axial bone metastasis is also presented. Written informed consent was obtained from the patient.

\section{Case report}

On July 4, 2014, a 73-year-old man was admitted to the Sir Run Run Shaw Hospital (Hangzhou, Zhejiang, China) due to pain and swelling of the distal phalanx of the left hallux, which had developed gradually over several months and progressed during the last 2 weeks prior to the patient's initial visit. The patient did not complain of any fever or changes in toe movement. The patient's medical history did not include any recent injuries. On November 28, 2012, the patient had been diagnosed with prostate cancer by prostate biopsy in Zhejiang Province People's Hospital (Hangzhou, Zhejiang, China), and in November 2013 the patient had been diagnosed with lung and vertebral bone metastases, as determined by positron emission tomography/computed tomography (PET/CT) and bone scans in the same hospital.

Due to the prostate cancer, the patient was treated with radioactive particle implantation in the prostate (iodine 125; $150 \mathrm{~Gy}$ ), endocrine therapy with casodex (50 mg 3 times a day; December 2, 2012-April 9, 2013) and goserelin acetate (3.6 mg subcutanoues once once every 28 days; Decemeber 2, 2012-April 9, 2013), chemotherapy with docetaxel in combination with prednisone (100 mg intraveous every 3 weeks; May 9, 2013-December 3, 2013), and endocrine therapy with abiraterone (1000 mg daily; January 14, 2014-July 4, 2014).

Upon the current admission, the physical examination did not reveal any abnormalities. The white blood cell, hemoglobin and platelet counts were 6,750 cells $/ \mathrm{mm}^{3}$ (normal range, 3,500-9,500 cells $\left./ \mathrm{mm}^{3}\right), 11.4 \mathrm{~g} / \mathrm{dl}(13.0-17.5 \mathrm{~g} / \mathrm{dl})$ and 110,000 cells $/ \mathrm{mm}^{3}$ (normal range, $100,000-300,000$ cells $/ \mathrm{mm}^{3}$ ), respectively. The patient had a high serum prostate-specific antigen (PSA) level ( $>10,000 \mathrm{ng} / \mathrm{ml}$; normal range, $<4 \mathrm{ng} / \mathrm{ml})$. An enhanced CT scan of the chest and abdomen showed a large 
Table I. Characteristics of patients with prostate cancer presenting with rare metastatic events.

\begin{tabular}{|c|c|c|c|c|c|}
\hline First author & Year & Age, years & Site & $\mathrm{PSA}, \mathrm{ng} / \mathrm{ml}$ & (Ref.) \\
\hline Massraf and Wand & 1998 & 62 & Thumb & 396,000 & (7) \\
\hline Patel et al & 2015 & 93 & Cutaneous; proximal right lower trunk & 415 & (8) \\
\hline Ogunmola et al & 2013 & 62 & Cutaneous; neck, limbs and trunk & Unknown & (9) \\
\hline Brown et al & 2014 & 73 & Cutaneous; left chest & 47 & (10) \\
\hline Keen and Hassan & 2013 & 70 & Cutaneous; left inguinal region and inner left thigh & 210 & (11) \\
\hline Martin et al & 2015 & 66 & Iris & 17 & (12) \\
\hline Soe et al & 2014 & 64 & Stomach and rectum & $>10,000$ & (13) \\
\hline Hélissey et al & 2015 & 87 & Parotid gland & 227 & (14) \\
\hline Zhang et al & 2014 & 76 & Proximal ureter & 18.14 & (15) \\
\hline Kaswala et al & 2014 & 42 & Duodenum & Unknown & (16) \\
\hline Wu et al & 2014 & 74 & Intramedullary spinal cord & Unknown & (17) \\
\hline Oliveira et al & 2012 & 73 & Larynx & 78 & (18) \\
\hline Freudlsperger et al & 2012 & 75 & Mandibular condyle & Unknown & (19) \\
\hline
\end{tabular}

PSA, prostate-specific antigen.

A
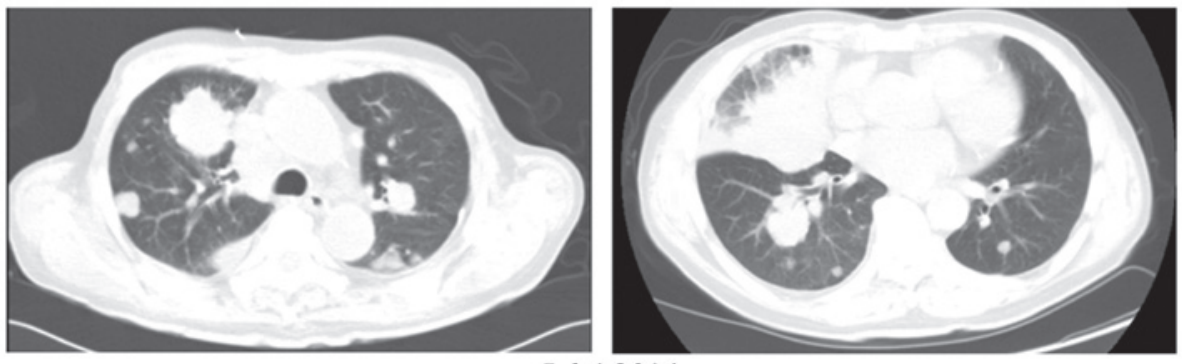

Jul 42014

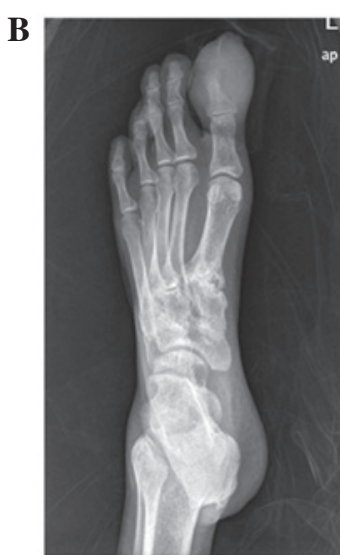

Jul 42014

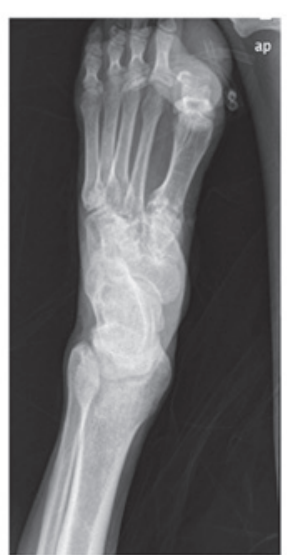

Jul 222014

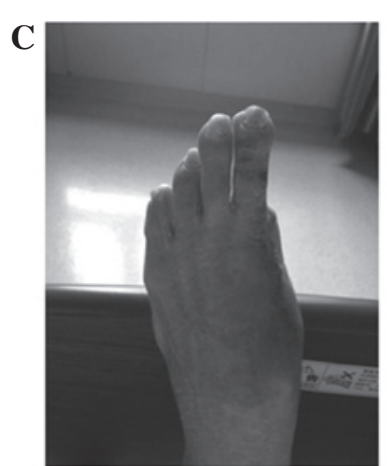

After the operation

Figure 1. Chest CT scan and X-ray findings. (A) Chest CT showing a large number of masses in each lung. (B) X-rays revealing the gradually developing morphological changes of the phalanx of the left hallux. (C) The patient's foot subsequent to amputation.

number of masses in each lung (Fig. 1A). As the patient was admitted due to a swollen distal phalanx of the left hallux, a bone scan or PET/CT scan was indicated, however, the patient refused. Therefore, an X-ray examination was performed (Fig. 1B).

The X-ray results showed morphological changes of the phalanx and surrounding soft-tissue swelling. Moreover, an obvious swelling was found on the terminal phalanx of the toe. The patient was first treated with incision and drainage of the soft tissue, but the swelling symptom could not be improved. On the contrary, it became more and more serious during the next 2 weeks, accompanied with osteolytic destruction (Fig. 1B). After an orthopedic consultation, an amputation of the phalanx of the left hallux was performed (Fig. 1C).

The patient was discharged a week after surgery. A follow-up visit every week was scheduled and no post-operative complications occurred. The resected specimen $(4 \mu \mathrm{m})$ was analyzed by histological (hematoxylin and eosin) and immunohistochemical 
$\mathrm{x} 10$

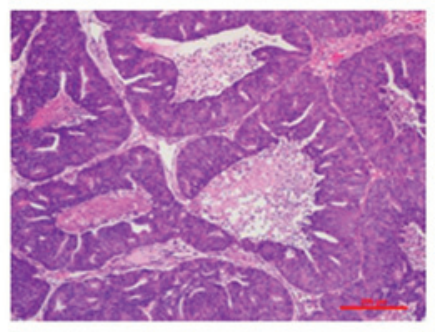

$\mathrm{H} \& \mathrm{E}$

P504S

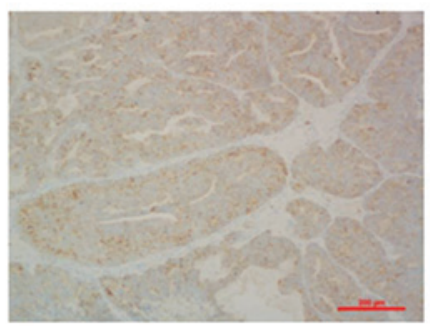

PSA

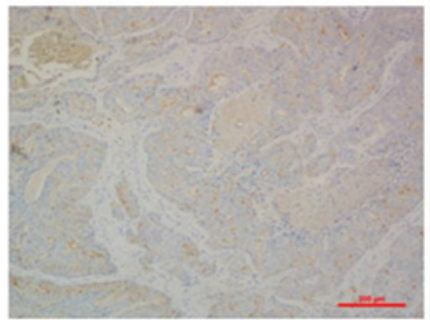

CK7

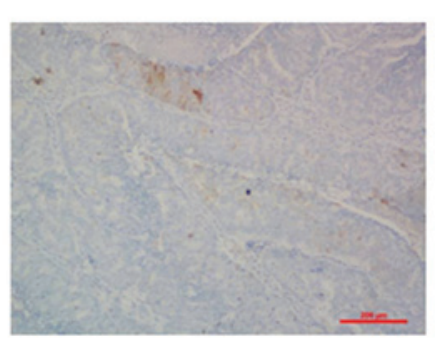

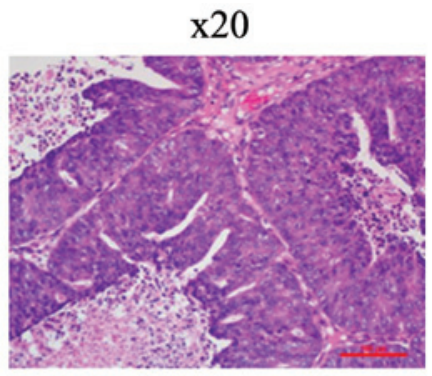
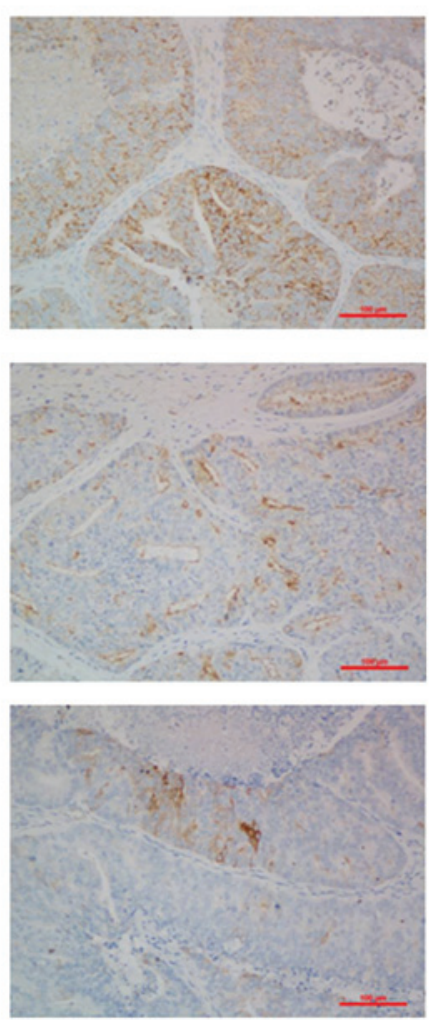

Figure 2. Pathological features with histological and immunohistochemical staining for P504S, PSA and CK7. Histological examination revealed a large island of tumor cells. Immunohistochemical staining revealed that the specimen cells were positive for P504S, PSA and CK7. PSA, prostate-specific antigen; CK7, cytokeratin 7; H\&E, hematoxylin and eosin.

staining. Notably, histological examination revealed a large island of tumor cells (Fig.2). Immunohistochemical analysis revealed that the tumor cells were positive for protein kinase P504S, PSA and cytokeratin (CK)7 (Fig.2), whereas the cells were negative for cluster of differentiation $(\mathrm{CD}) 56$, synaptophysin (Syn), chromogranin A (CgA) and neuron-specific enolase (NSE). The following antibodies were used: Mouse monoclonal anti-P504S (catalog number, sc-81710; dilution, 1:500; Santa Cruz Biotechnology, Inc., Dallas, TX, USA), mouse monoclonal anti-PSA (catalog number, sc-7316; dilution, 1:500; Santa Cruz Biotechnology, Inc.), mouse monoclonal anti-CK7 (catalog number, ab-9021; dilution, Abcam, Cambridge, MA, USA), mouse monoclonal anti-CD56 (catalog number, 3576; dilution, 1:100; Cell Signaling Technology, Inc., Danvers, MA, USA), mouse monoclonal anti-Syn (catalog number, sc-398849; dilution, 1:500; Santa Cruz Biotechnology, Inc.), rabbit polyclonal anti-CgA (catalog number, ab-15160; dilution, 1:1,000; Abcam) and rabbit polyclonal anti-NSE (catalog number, ab-53025; dilution, 1:1,000; Abcam). Taken together, these analyses confirmed that the lesion of the distal phalanx of the left hallux was a metastatic mass from prostate cancer.
Based on the aforementioned evidence, a diagnosis of prostate cancer with metastasis to the distal phalanx of the left hallux was made. The patient succumbed to cardiac and respiratory failure on February 15, 2015.

\section{Discussion}

Prostate cancer is the most common noncutaneous cancer in men around the world and it preferentially spreads to the skeleton. Therefore, bone is the dominant and often only metastatic lesion for the vast majority of men with advanced prostate cancer (5). Bone metastasis is the main cause for the morbidity and mortality associated with prostate cancer, leading to skeletal-related events that include severe pain, pathological fractures and the risk of spinal cord compromise (6). The non-axial bone metastasis of prostate cancer is an extremely rare event. Only phalangeal thumb metastasis has been reported to date (7). Besides bone, lymph node, lung, liver, pleura and adrenal gland metastases, several rare metastatic events have been reported in prostate cancer patients. Although cutaneous metastasis from prostate cancer is rare in the literature, it has recently been 
reported in a large number of the case studies (Table I) (8-11). In addition, the iris (12), gastrointestinal tract (13), parotid gland (14), proximal ureter (15), duodenum (16), intramedullary spinal cord (17), larynx (18) and mandibular condyle (19) are also involved metastatic sites of prostate cancer. Nonetheless, prostate cancer with metastasis to the phalanx of the toe has been not reported to date. The present case is therefore the first report on this rare type of metastasis.

Several tumor markers are useful in determining the diagnosis of prostate cancer. PSA is a specific tumor marker for prostate cancer that commonly occurs at high serum levels in affected patients (20). PSA evaluation by immunohistochemistry has been demonstrated to be a useful tool for determining the diagnosis and prognosis of prostate cancer (21). However, PSA immunohistochemistry for the detection of metastatic prostate cancer can also be of limited utility (22). In the present case, the immunoreactivity of PSA staining tended to be mildly positive in the metastatic site.

$\alpha$-methylacyl-CoA racemase (AMACR/P504S) is a metabolic enzyme whose overexpression has been shown to be a diagnostic indicator of prostatic adenocarcinoma in clinical practice $(23,24)$. Increasing evidence has shown AMACR/P504S is a diagnostically useful marker for prostatic cancer $(25,26)$. In the present study, immunohistochemical examinations revealed positive P504S staining in the surgical sample. CK7 is commonly used to identify primary tumor sites and to distinguish invasive prostate cancer from cancer in situ (27). However, the immunohistochemical examination of $\mathrm{CK}$ is not specific for prostatic cancer. Focal positivity for CK7 was found in the present results.

The neuroendocrine antigens (NSE, CgA, Syn and CD56) are useful in identifying neuroendocrine prostate cancer, but are usually not expressed in prostatic adenocarcinoma (28). In agreement with this, the present immunohistochemical staining also showed negative results for CD56, Syn, CgA and NSE in prostatic adenocarcinoma.

In summary, the present study is the first to report a case of prostate cancer metastasis to the phalanx of the toe, in a man that was referred because of persistent pain and swelling of the distal phalanx of the left hallux, initially misdiagnosed and treated as abscess. No post-surgery complications and recurrence occurred following local surgery. The observations made in the present study contribute to a better understanding of bone metastasis of prostate cancer.

\section{Acknowledgements}

This study was supported by grants from the National Natural Science Foundation of China (no. 81301891), the Zhejiang Provincial Natural Science Foundation of China (no. LQ13H160008), the Zhejiang Province Science and Technology Project of Traditional Chinese Medicine (no. 2015ZB033) and the Zhengshu Medical Elite Scholarship Fund.

\section{References}

1. Siegel RL, Miller KD and Jemal A: Cancer statistics, 2015. CA Cancer J Clin 65: 5-29, 2015.

2. Merabishvili VM, Petrova NG, Atroshchenko AV and Kharitonov MV: Epidemiology of prostate cancer (cohort study). Vopr Onkol 60: 457-463, 2014 (In Russian).
3. Toomey A and Friedman L: Mortality in cancer patients after a fall-related injury: The impact of cancer spread and type. Injury 45: 1710-1716, 2014.

4. Cho JY, Shim EJ, Kim IS, Nam EM, Choi MY, Lee KE, Mun YC, Seoung CM, Lee SN, Song DE and Han WS: Cancer of unknown primary finally revealed to be a metastatic prostate cancer: A case report. Cancer Res Treat 41: 45-49, 2009.

5. Tu SM, Millikan RE, Mengistu B, Delpassand ES, Amato RJ, Pagliaro LC, Daliani D, Papandreou CN, Smith TL, Kim J, et al: Bone-targeted therapy for advanced androgen-independent carcinoma of the prostate: A randomised phase II trial. Lancet 357: 336-341, 2001.

6. Suzman DL, Boikos SA and Carducci MA: Bone-targeting agents in prostate cancer. Cancer Metastasis Rev 33: 619-628, 2014

7. Massraf $\mathrm{AB}$ and Wand JS: Haemorrhagic secondary prostatic metastasis of the terminal phalanx of the thumb. Injury 29: 243-245, 1998.

8. Patel P, Patel J and Siddiqui S: Recurrence of prostate cancer with cutaneous metastasis after radical prostatectomy. Case Rep Urol 2015: 825175, 2015.

9. Ogunmola AO, Shittu OB and Olapade-Olaopa EO: Cutaneous metastasis from prostate cancer in a nigerian: A case report and literature review. Afr J Med Med Sci 42: 283-286, 2013.

10. Brown GT, Patel V and Lee CC: Cutaneous metastasis of prostate cancer: A case report and review of the literature with bioinformatics analysis of multiple healthcare delivery networks. J Cutan Pathol 41: 524-528, 2014.

11. Keen MA and Hassan I: Cutaneous metastasis of an advanced prostate cancer. Indian J Dermatol Venereol Leprol 79: 828-829, 2013.

12. Martin V, Cuenca X, Lopez S, Albertini AF, Lang P, Simon JM, Hémery CG, Feuvret L and Mazeron JJ: Iris metastasis from prostate carcinoma: A case report and review of the literature. Cancer Radiother 19: 331-333, 2015.

13. Soe AM, Bordia S, Xiao PQ, Lopez-Morra H, Tejada J, Atluri S and Krishnaiah M: A rare presentation of metastasis of prostate adenocarcinoma to the stomach and rectum. J Gastric Cancer 14: 271-274, 2014.

14. Hélissey C, Rouanne M, Arnaud FX and Le Moulec S: Parotid gland metastasis from prostate cancer: Is docetaxel still the best treatment option? Anticancer Drugs 26: 367-370, 2015.

15. Zhang T, Wang Q, Min J, Yu D, Xie D, Wang Y, Ding D, Chen L, Zou C, Zhang Z and Wang D: Metastasis to the proximal ureter from prostatic adenocarcinoma: A rare metastatic pattern. Can Urol Assoc J 8: E859-E861, 2014.

16. Kaswala DH, Patel N, Jadallah S and Wang W: Metastatic prostate cancer to the duodenum: A rare case. J Family Med Prim Care 3: 166-168, 2014.

17. Wu Z, Xu S, Zhong C, Gao Y, Liu Q, Zheng Y, Guo Y, Wang Y, Luo Q and Jiang J: Intramedullary conus medullaris metastasis from prostate carcinoma: A case report and review of the literature. Oncol Lett 7: 717-720, 2014.

18. Oliveira JA, Said Rde A, Cartaxo Rde S, Santos JA and Gondim RL: Laryngeal metastasis of a prostate carcinoma: One rare entity. Braz J Otorhinolaryngol 78: 135, 2012 (In Portuguese).

19. Freudlsperger C, Kurth R, Werner MK, Hoffmann J and Reinert S: Condylar metastasis from prostatic carcinoma mimicking temporomandibular disorder: A case report. Oral Maxillofac Surg 16: 79-82, 2012.

20. Bhavsar T, McCue P and Birbe R: Molecular diagnosis of prostate cancer: Are we up to age? Semin Oncol 40: 259-275, 2013.

21. Garde SV, Sheth AR, Venkatesan VM, Panchal CJ, Porter AT and Grignon DJ: Prostate inhibin peptide (PIP) in prostate cancer: A comparative immunohistochemical study with prostate-specific antigen (PSA) and prostatic acid phosphatase (PAP). Cancer Lett 78: 11-17, 1994.

22. Bernacki KD, Fields KL and Roh MH: The utility of PSMA and PSA immunohistochemistry in the cytologic diagnosis of metastatic prostate carcinoma. Diagn Cytopathol 42: 570-575, 2014.

23. Wilson BA, Wang H, Nacev BA, Mease RC, Liu JO, Pomper MG and Isaacs WB: High-throughput screen identifies novel inhibitors of cancer biomarker $\alpha$-methylacyl coenzyme A racemase (AMACR/P504S). Mol Cancer Ther 10: 825-838, 2011.

24. Kumaresan K, Kakkar N, Verma A, Mandal AK, Singh SK and Joshi K: Diagnostic utility of $\alpha$-methylacyl CoA racemase (P504S) \& HMWCK in morphologically difficult prostate cancer. Diagn Pathol 5: 83, 2010. 
25. Shen Y, Wang Z, Zhu J, Chen Y, Gu W and Liu Q $\alpha$-Methylacyl-CoA racemase (P504S) is a useful marker for the differential diagnosis of solid pseudopapillary neoplasm of the pancreas. Ann Diagn Pathol 18: 146-150, 2014.

26. Murray NP,Calaf GM, Badinez L, Dueñas R, Badinez O, Orellana N, Reyes E and Fuentealba C: P504S expressing circulating prostate cells as a marker for prostate cancer. Oncol Rep 24: 687-692, 2010.
27. Fichtenbaum EJ, Marsh WL Jr and Zynger DL: CK5, CK5/6, and double-stains CK7/CK5 and p53/CK5 discriminate in situ vs. invasive urothelial cancer in the prostate. Am J Clin Pathol 138: 190-197, 2012.

28. Aggarwal R, Zhang T, Small EJ and Armstrong AJ: Neuroendocrine prostate cancer: Subtypes, biology, and clinical outcomes. J Natl Compr Canc Netw 12: 719-726, 2014. 